\title{
Of Mice and Men: Defining the Role of Interleukin 17 in Rheumatoid Arthritis
}

In a recent issue of The Journal, Pavelka and colleagues reported on a negative study of brodalumab, the interleukin 17 (IL-17) inhibitor, in rheumatoid arthritis (RA) ${ }^{1}$. In addition to the authors' conclusion that there is no reason to pursue further evaluation of the molecule, an antibody against the IL-17 receptor A (IL-17RA), in this disease, there are a number of other important lessons to be learned from this publication. Combined with previously published data for secukinumab $^{2}$ and ixekizumab ${ }^{3}, 2$ anti-IL-17A antibodies, the results of this study strongly suggest that the IL-17 pathway is not an appropriate target in RA. This conclusion comes despite both animal data suggesting potential benefit and clinical evidence that IL-17 inhibition is effective in psoriatic arthritis (PsA). The latter observation is instructive, and may provide a clue to important pathogenic differences between 2 outwardly similar forms of inflammatory arthritis.

The study itself compared 3 different dose regimens of brodalumab (70 mg, $140 \mathrm{mg}$, and $210 \mathrm{mg}$ ) and placebo; doses were given every 2 weeks, with an additional loading dose at 1 week. The population studied was a typical methotrexate (MTX) inadequate responder population ( $\sim 80 \%$ female, $7-8$ years of disease, Disease Activity Score $28 \sim 6.4$, Health Assessment Questionnaire-Disability Index 1.4, and mean dose of MTX $17 \mathrm{mg} /$ week). The primary endpoint selected was the ACR50 (American College of Rheumatology 50\% improvement) response rate at Week 12 . There was no benefit seen for any of the brodalumab doses over placebo, and no dose response seen for the 3 tested doses. None of the secondary endpoints were achieved either. This was in contrast to the brodalumab data in PsA, where doses of 140 $\mathrm{mg}$ and $280 \mathrm{mg}$ were both effective. On the positive side, there were no signals of specific adverse events (AE) or serious adverse events (SAE) with brodalumab in the RA trial, and withdrawals for $\mathrm{AE}$ were similar across the 4 groups.

In a previously published study of secukinumab in 237 MTX inadequate responders with RA, the primary endpoint of ACR20 response at Week 16 was not met ${ }^{2}$. There was also no statistically significant difference from placebo for any of the secondary endpoints, although there was numerical improvement compared with placebo for several of these secondary endpoints. The authors of this study were a bit more forgiving. They suggested that the trends toward response identified suggested a signal of response that warrants further study, perhaps to identify biomarkers of response to IL-17A inhibition.

In a study of ixekizumab, another IL-17A antibody, 5 doses of the antibody were examined in 448 patients, 260 of whom were biologic naive and 188 of whom were tumor necrosis factor (TNF) inhibitor inadequate responders ${ }^{3}$. The doses were similar, but not identical, to the doses studied in psoriasis trials with ixekizumab, and only the 2 highest doses were studied in the latter group ${ }^{4,5}$. This study used a complicated primary endpoint, the dose-response relationship for ixekizumab in the ACR20 response at 12 weeks, and this was met with a $\mathrm{p}$ value of 0.031 . However, only the middle of the 5 escalating dose groups ( $30 \mathrm{mg}$ every 2 weeks) actually had an ACR20 response that was statistically greater than placebo at 12 weeks. Most of the ixekizumab dose groups had an ACR50 response rate that was greater than placebo ( $\mathrm{p}<0.05$ ), but the average rate of response was only 20-30\%, compared with $9 \%$ for placebo. The 2 doses tested for the TNF inadequate responders generated an ACR20 response that was statistically better than placebo $(\mathrm{p}<0.05)$, but the difference in response rate was $<20 \%$.

Despite these rather modest responses, the authors generously concluded that ixekizumab produced a rapid and significant response in both biologic naive and TNF inhibitor inadequate responder patients, and that this molecule might represent a viable treatment alternative. They did suggest that the fact that brodalumab targets the IL-17 receptor, while ixekizumab (and secukinumab) target the cytokine itself, might explain some of the differences in the trial results. As with brodalumab, the safety profile of secukinumab and ixekizumab in their respective trials was good, with no signals for specific AE or SAE with either agent.

See Brodalumab in RA, June 2015 issue, page 912

Personal non-commercial use only. The Journal of Rheumatology Copyright @ 2015 . All rights reserved. 
Two other agents that target the IL-17 pathway, ustekinumab, an IL-12/23 antibody, and guselkumab, an IL-23 antibody, have been studied in a trial of MTX inadequate responders in RA (IL-23 drives Th17 polarization and thus IL-17 production). In this study, which has been reported on clinicaltrials.gov but has not yet been published, neither antibody was significantly more effective than placebo at achieving the primary endpoint of ACR20 response at 28 weeks ${ }^{6}$. In sum, despite the rather positive spin by the authors of the secukinumab and ixekizumab studies, the weight of the evidence suggests that the IL-17 pathway is simply not an attractive target in RA, especially in an era of other highly effective medications. As the authors of the secukinumab trial suggest, the availability of specific biomarkers predicting response might change this conclusion, but such biomarkers have yet to be identified.

What can we learn from such negative studies? It must be noted that none of the antibodies studied are ineffective molecules. Indeed, all 5 of the antibodies above have been demonstrated to be effective in skin psoriasis, and 2 (ustekinumab and secukinumab) have been approved in the United States for this indication ${ }^{4,5,7,8,9}$. Ustekinumab has also been approved for the treatment of PsA, and there have been studies demonstrating the effectiveness of brodalumab and secukinumab in this disease as well ${ }^{10,11}$.

What, then, might explain the differences in the efficacy of IL-17 pathway inhibition between RA and PsA? There has certainly been evidence pointing toward a role for IL-17 in RA pathogenesis. There is enhanced production of IL-17 by Th17 cells in the disease, and IL-17 itself enhances the production of a number of inflammatory mediators, as well as the survival and proliferation of synoviocytes and immune cells $^{12}$. IL-17 acts independently of TNF and IL-1 in humans, which might suggest a therapeutic role for its inhibition ${ }^{13}$. In animal models, and in humans, there is evidence that both Th17 cells and the IL-17 they produce contribute to cartilage and bone damage ${ }^{14,15}$. In fact, intraarticular injections of IL-17 generate joint damage in murine knees ${ }^{16}$. Nevertheless, animal models are just that; they provide clues to the mechanisms of human disease, but they are not the same thing. In this case, although the mouse models may provide insight into RA pathogenesis, it seems likely that there are differences in the role that Th17 cells and IL-17 signaling play in mice and men $^{17}$. In PsA, on the other hand, the IL-17 pathway seems to be playing a more central role in the inflammatory arthritis.

While disappointing, the results of the trial by Pavelka, et $a l^{1}$ are important observations, and the authors are to be commended for reporting them. Much has been written about the importance of publishing negative clinical trial data ${ }^{18}$. Doing so gets this information into the public domain, so that others have access to it. It allows for critical peer review of the data, to determine whether the results are truly negative, or perhaps merely underpowered to identify a positive result.
Having the negative results in the public domain also ensures that future metaanalyses can provide a balanced and accurate evaluation.

Finally, the availability of negative results such as these helps to ensure that further effort is not wasted pursuing targets that show little promise for therapy. At the same time, it may inspire more investigation into why the results were negative, potentially generating new insights into disease pathogenesis. In this case, the apparent lack of efficacy of IL-17 inhibition in RA, contrasted with its apparent efficacy in PsA, may inform further research into the unique and distinguishing cellular and molecular aspects of these 2 inflammatory arthritides. Much has been made of the benchto- bedside successes achieved by the biologic therapies in rheumatic diseases. Data from this study may help to drive more research back at the bench, so that we may better understand the diseases themselves.

ERIC M. RUDERMAN, MD,

Northwestern University Feinberg School of Medicine, Division of Rheumatology,

675 North St. Clair, Suite 14-100

Chicago, Illinois 60611, USA.

Address correspondence to Dr. Ruderman.

E-mail: e-ruderman@northwestern.edu

\section{REFERENCES}

1. Pavelka K, Chon Y, Newmark R, Lin SL, Baumgartner S, Erondu N. A study to evaluate the safety, tolerability, and efficacy of brodalumab in subjects with rheumatoid arthritis and an inadequate response to methotrexate. J Rheumatol 2015;42:912-9.

2. Genovese MC, Durez P, Richards HB, Supronik J, Dokoupilova E, Mazurov V, et al. Efficacy and safety of secukinumab in patients with rheumatoid arthritis: a phase II, dose-finding, double-blind, randomised, placebo controlled study. Ann Rheum Dis 2013;72:863-9

3. Genovese MC, Greenwald M, Cho CS, Berman A, Jin L, Cameron GS, et al. A phase II randomized study of subcutaneous ixekizumab, an anti-interleukin-17 monoclonal antibody, in rheumatoid arthritis patients who were naive to biologic agents or had an inadequate response to tumor necrosis factor inhibitors. Arthritis Rheumatol 2014;66:1693-704.

4. Gordon KB, Leonardi CL, Lebwohl M, Blauvelt A, Cameron GS, Braun D, et al. A 52-week, open-label study of the efficacy and safety of ixekizumab, an anti-interleukin-17A monoclonal antibody, in patients with chronic plaque psoriasis. J Am Acad Dermatol 2014;71:1176-82.

5. Leonardi C, Matheson R, Zachariae C, Cameron G, Li L, Edson-Heredia E, et al. Anti-interleukin-17 monoclonal antibody ixekizumab in chronic plaque psoriasis. N Engl J Med 2012;366:1190-9.

6. A study of the effectiveness and safety of ustekinumab (STELARA) and CNTO 1959 administered under the skin of patients with active rheumatoid arthritis, despite existing methotrexate therapy. [Internet. Accessed May 22, 2015.] Available from: https:// clinicaltrials.gov/ct2/show/NCT01645280

7. Langley RG, Elewski BE, Lebwohl M, Reich K, Griffiths CE, Papp $\mathrm{K}$, et al. Secukinumab in plaque psoriasis - results of two phase 3 trials. N Engl J Med 2014;371:326-38.

8. Tausend W, Downing C, Tyring S. Systematic review of interleukin-12, interleukin-17, and interleukin-23 pathway inhibitors

Personal non-commercial use only. The Journal of Rheumatology Copyright $\subset$ $\subset$ 2015. All rights reserved 
for the treatment of moderate-to-severe chronic plaque psoriasis: ustekinumab, briakinumab, tildrakizumab, guselkumab, secukinumab, ixekizumab, and brodalumab. J Cutan Med Surg 2014;18:156-69.

9. Papp KA, Leonardi C, Menter A, Ortonne JP, Krueger JG, Kricorian $\mathrm{G}$, et al. Brodalumab, an anti-interleukin-17-receptor antibody for psoriasis. N Engl J Med 2012;366:1181-9.

10. McInnes IB, Kavanaugh A, Gottlieb AB, Puig L, Rahman P, Ritchlin C, et al. Efficacy and safety of ustekinumab in patients with active psoriatic arthritis: 1 year results of the phase 3 , multicentre, double-blind, placebo-controlled PSUMMIT 1 trial. Lancet 2013;382:780-9

11. Ritchlin C, Rahman P, Kavanaugh A, McInnes IB, Puig L, Li S, et al. Efficacy and safety of the anti-IL-12/23 p40 monoclonal antibody, ustekinumab, in patients with active psoriatic arthritis despite conventional non-biological and biological anti-tumour necrosis factor therapy: 6-month and 1-year results of the phase 3 , multicentre, double-blind, placebo-controlled, randomised PSUMMIT 2 trial. Ann Rheum Dis 2014;73:990-9.

12. Benedetti $\mathrm{G}$, Miossec P. Interleukin 17 contributes to the chronicity of inflammatory diseases such as rheumatoid arthritis. Eur J Immunol 2014;44:339-47.
13. Lubberts E, Koenders MI, van den Berg WB. The role of T-cell interleukin-17 in conducting destructive arthritis: lessons from animal models. Arthritis Res Ther 2005;7:29-37.

14. Pollinger B. IL-17 producing T cells in mouse models of multiple sclerosis and rheumatoid arthritis. J Mol Med 2012;90:613-24.

15. Li X, Yuan FL, Lu WG, Zhao YQ, Li CW, Li JP, et al. The role of interleukin-17 in mediating joint destruction in rheumatoid arthritis. Biochem Biophys Res Commun 2010;397:131-5.

16. Dudler J, Renggli-Zulliger N, Busso N, Lotz M, So A. Effect of interleukin 17 on proteoglycan degradation in murine knee joints Ann Rheum Dis 2000;59:529-32.

17. Corneth OB, Mus AM, Asmawidjaja PS, Klein Wolterink RG, van Nimwegen M, Brem MD, et al. Absence of interleukin-17 receptor a signaling prevents autoimmune inflammation of the joint and leads to a Th2-like phenotype in collagen-induced arthritis. Arthritis Rheumatol 2014;66:340-9.

18. Sandercock P. Negative results: why do they need to be published? Int J Stroke 2012;7:32-3.

J Rheumatol 2015;42:1069-71; doi:10.3899/jrheum.150554 